\title{
Amin Maalouf ou la diversité salvatrice
}

\author{
Dr.Amany Ibrahim Ghander
}

Venu à la littérature par le journalisme, l'œuvre de Maalouf expose ses opinions politiques ainsi que ses positions sociologico-linguistiques. C'est uneillustration de son implication dans le débat sur les diversités linguistiques, culturelles et identitaires, sur le dialogue possible ou pas, entre le Nord et le Sud, de même que sur l'harmonie, envisageable ou chimérique, entre les communautés.

Malgré l'aspect protéiforme ${ }^{1}$ de son œuvre, Maalouf affirme la continuité de son parcours ${ }^{2}$. Au lieu de suivre une carrière diplomatique ou politique, il a toujours choisi l'écriture. Son œuvre est un hymne à l'humanisme. Sa détermination àdéfendre la pluralité culturelle, religieuse et politique hante son esprit tout au long de son itinéraire. Cette voie lui a ouvert de vastes horizons sur sa carrière d'écrivain et sur sa vision du monde.

Publié en 1983, Les croisades vues par les Arabes, est son premier livrehistorico-littéraire oùil assoit les thèmesrécurrents qui ne cesseront de revenir dans ses romans et ses essais. Maalouf y pose les prémices de la dichotomie Orient-Occident, dichotomie qui ne cessera de se manifester de multiples façons au cours de sièclesà venir depuis «les guerres entre Ottomans et Hasbourg, l'orientalisme et les nombreux conflits moyensorientaux jusqu'à la croisade contre le mal de George W. Bush et le djihadisme actuel. $»^{3}$

\footnotetext{
${ }^{1}$ L'œuvre de Maalouf comprend des essais sociopolitiques Les Identités meurtrières(1998) et Le dérèglement du monde(2009)), un récit-essai historique La croisade vues par les Arabes(1993), plusieurs romans historiques dont Léon l'Africain(1986), Samarcande(1988), Le Rocher de Tanios (1993) et Le périple de Baldassare (2000)), un récit autobiographique Origines (2004), un récit de science-fiction Le premier siècle après Beatrice (1992), des livrets d'opéra L'Amour de loin(2001), Adriana Mater(2004), La passion de Simone(2006) et Émilie (2010)) et un roman Les Désorientés(2012).

2 Cf. BOUVET, Rachel et EL KETTANI, Soundous, Amin Maalouf. Une cuvre à revisiter, Presse Universitaire du Québec, 2014, p.261.

${ }^{3}$ Ibid., p. 152.
} 


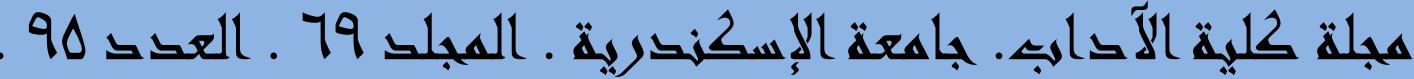

Cet ouvrage,comme le titre l'indique, offre le point de vue arabe et oriental, autrement dit le point de vue de l'autre, à un lectorat essentiellementoccidental. Et c'est justement là que réside tout l'apport d'Amin Maalouf à la littérature età la pensée : un point de vue différent qui résulte d'un «déplacement de perspective »" C'est aussi le cas de Léon l'Africain où la chute de Grenade est décrite selon le point de vue des arabes.

Or ce qui permet à Maalouf d'opérer ce déplacement de perspective c'est bien sa situation d'écrivain en exil qui le rend capable de considérer les problèmes sociaux et identitaires à partir d'une position en retrait : «Il offre à la fois une perspective du dehors, provenant de sa situationexcentrée et une approche d'empathie multilatérale issue de sa profonde compréhension des identités plurielles et de l'impossibilité de l'unicité identitaire. »5

Maalouf a également la particularité de rester toujours impartial à l'égard de toutes les cultures, les religions ou les civilisations.Il a le courage de dire ce qu'il pense, contre vents et marées, renvoyant dos à dos l'Orient et l'Occident.

Son projet d'écriture se fondeaussi sur une vision de l'histoire car pour lui l'Histoire est «un réservoir de mondes possibles de la pluralité identitaires et de l'universalisme. ${ }^{6}$

Ainsi, dansLes jardins de lumière, Maalouf remonte à Mani, le fondateur du manichéisme, religion caractéristique par sapenséetolérante et humaniste. Il se demande comment de toute la religion de Mani, sa foi généreuse et son message d'harmonie et de tolérance, il ne reste plus rien. Comment nous n'avons gardé que des mots «manichéen» ou «manichéisme » devenus comme des insultes. Il tente de mettre en valeur le caractère tolérant et humaniste de ce grand messager de la Perse, lors de l'apogée de la dynastie sassanide. La fin dramatique de Mani n'est, en effet, qu'un résultat du triomphe du fanatisme.

A travers la biographie de Léon l'Africain, ce caractère humaniste et fascinant de la Renaissance, Maalouf montre les valeurs sublimes de l'humanisme. Il tend à reconstituer les périples de ce personnage

\footnotetext{
${ }^{4}$ VOLTERRANI, Egi, « Autobiographie a deux voix », entretien d’Amin Maalouf avec Egi Volterrani, réalisé en décembre 2001, http://aminmaalouf.net/fr/sur-amin/autobiographie-a-deux-voix/, consulté le 3 mars 2018.

${ }^{5}$ BOUVET, Rachel et EL KETTANI, Soundous, Op.cit., p. p.152, 153.

${ }^{6}$ Ibid., p. 179.
} 


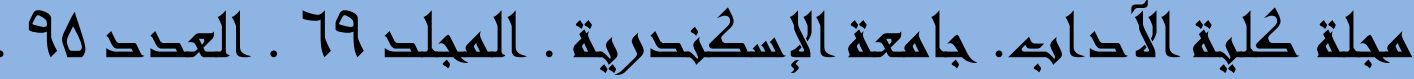

exceptionnel, à une période florissante de l'histoire du XVIe siècle. Ce choixingénieux des personnages a permis à Maalouf de faire ressortir leur esprit humaniste, ou plutôt, son esprit humaniste, qu'il résume en une valeur : la dignité de l'être humain, de tout être humain.

Ainsi, Maalouf redonne-t-il au terme humanisme sa valeur authentique, tel qu'il a été appréhendé par la Renaissance et le siècle des Lumières, ce terme qui, malheureusement, ne résonne plus de la même manière dans le monde d'aujourd'hui.

Mais c'est surtout dans ses deux essais, Les Identitésmeurtrières (1998) et Le Dérèglement du monde(2009)que Maalouf livre l'essentiel de sa pensée. Ces deux essais, en fait, reprennent les mêmesidéesdéveloppées dans ses romans, mais dans une approche actuelle, touchant de près les questions de notre temps.

Toute la pensée de Maalouf part de cette constatation :

«Nous sommes passés d'un monde où les clivages étaient principalement idéologiques et où le débatétait incessant, à un monde où les clivages sont principalement identitaires et où il y a peu de place pour le débat. Chacun proclame ses appartenances à la face des autres, lance ses anathèmes, mobilise les siens, diabolise ses ennemis $q u$ 'y aurait-il d'autre à dire ? ${ }^{7}$

C'est cette frénésie identitaire qui l'inquiète. Attentif au moindre problème, Maalouf analyse tour àtour les questions brûlantes qui secoue le monde. Il est vrai que les débats et les écritsgéopolitiques ne manquent pas maisMaaloufconçoit la problématiqueà travers une approche qui lui est propre.

Les Identitésmeurtrières, en fait, a été publié en 1998 aux Éditions Grasset. Vingt ans après, l'actualité donne encore plus de poids à cet essai prémonitoire sur lequel nous nous baserons pour analyser l'essentiel de la pensée de Maalouf.

En effet, dans cet essai Maalouf essayait de comprendre pourquoi tant de personne commettent tant de crimes au nom de leur identité religieuse,

\footnotetext{
${ }^{7}$ Le Dérèglement du monde, Paris, Grasset \& Fasquelle, 2009, p.23.
} 


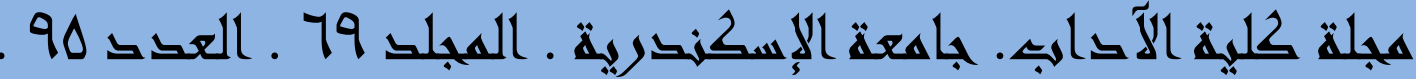

ethnique, nationale ou autre. Le danger dont il parle, nous le comprenons encore mieux aujourd'hui.

Dans son analyse des déchainements identitaires, Maalouf se base sur son expérience personnelle. En effet, dès le début de l'éclatement de la guerre libanaise, il refusa de prendre position dans les conflits qu'il jugeait absurde et suicidaire. En 1976, ildébarqua 'volontairement'" à Paris, outré de voir son pays sombrer dans la violence et la guerre civile. Il choisit le chemin de l'exil et fut contraint de refaire sa vie de fond en comble. Cette situation d'immigré, « d'êtreirrémédiablementminoritaire, irrémédiablementétranger », lui a permis de jouer pleinement son rôle de «passeur de cultures $»^{8}$, de chantre des exilés et d'ambassadeur des immigrants.Il revendique ce rôle de médiateur entre l'Orient et l'Occident, prêche pour un monde du multiculturalisme et de l'identité multiple, au point de se voir confier en 2007 la présidence du groupe des intellectuels pour le dialogue interculturel constitué par la Commission européenne.

L'humanisme est le cheval de bataille de Maalouf. C'est la toile de fond à partir de laquelle il n'a cessé, depuis plus de troisdécades d'ajuster son regard sur les cultures pour mieux apprendre à respecter les différences et les particularités de tous.

Dans notre approche de la pensée de Maalouf, il nous a semblé découvrir quatre axes principaux autour desquelles gravite toute son œuvre, voire sa pensée : le problème identitaire, le problème de l'immigration, le problème de l'islam face à la modernité et la question de la mondialisation.

Le premier axe est bien celui de l'identité. Dans Les Identitésmeurtrières, Maalouf commence par «décortiquer » l'identité, non point celle de notre carte d'identité car évidemment ce n'est point celle-ci qui dit qui nous sommes, mais notre identité complexe qui fait que chacun de nous est unique.

Appartenant à plusieurs cultures, Maalouf s'interroge sur la construction de l'identité. Pourquoi les hommes ont-ils du mal à gérer leurs diverses appartenances ? Pourquoi recourir à la violence pour affirmer son identité ?

L'essai s'ouvre sur une question classique que tout immigré redoute dans ses discussions, soit avec les autochtones, soit avec des immigrés

${ }^{8}$ VOLTERRANI, Egi, Art.cit. 


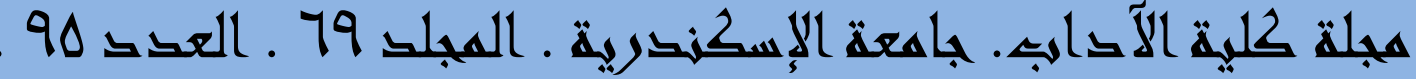

comme lui. «Êtes-vousplutôt libanais ou plutôtfrançais? » Et sa réponse vient chaque fois la même. Il est à la fois libanais et français. Ceci est bien le propre de l'identité selon lui, conception dont il est lui-même lemodèle. "Serais-je plus authentique si je m'amputais d'une partie de moimême? ${ }^{9}$

«Moitiéfrançais, donc, et moitiélibanais ? Pas du tout! l'identité ne se compartimente pas, elle ne se répartit ni par moitiés, ni par tiers, ni par plages cloisonnées. Je n'ai pas plusieursidentités, j'en ai une seule, faite de tous les éléments qui l'ont façonnée selon un "dosage" particulier qui n'est jamais le même d'une personne à l'autre. » ${ }^{10}$

Maalouf se démarque de toute appartenance spécifique. Il ne renie point la religion de ses pères, ni son attachement à son pays d'origine, ni l'influence de sa langue maternelle:l'arabe. Mais pour lui, l'identité de chaque personne est constituée d'une foule d'éléments. Il y a bien l'appartenanceà une religion, une nationalité, parfois deux, un groupe ethnique ou linguistique, une profession, un certain milieu social... et ainsi de suite. Tel que le formule Maalouf, «la liste est [...], virtuellement illimitée. » ${ }^{11}$

D'autre part, ces appartenances n'ont pas la même importance, d'ailleurs pas au même moment. "L'identité n'est pas donnée une fois pour toutes, elle se construit et se transforme tout au long de l'existence. ${ }^{12}$ Elle change avec le temps et modifie profondément nos comportements. Il est vrai que parfois les gens choisissent de mettre en avant une appartenance qu'ils considèrent majeure ou supérieure. Ils appellent celaleur identité bien que la vérité est beaucoup plus complexe.

«Si chacun de ces éléments peut se rencontrer chez un grand nombre d'individus, jamais on ne retrouve la même combinaison chez deux

\footnotetext{
${ }^{9}$ Les Identités meurtrières, Grasset, 2017, (1ère édition 1998), p.7.

${ }^{10}$ Ibid., p.8.

${ }^{11}$ Ibid., p.17.

${ }^{12}$ Ibid., p.31.
} 


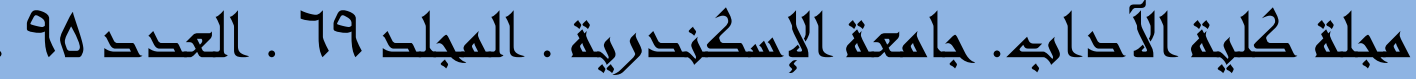

personnes différentes, et c'est justement cela qui fait la richesse de chacun, sa valeur propre, c'est ce qui fait que tout être est singulier et potentiellement irremplaçable. ${ }^{13}$

Ainsi, lorsque Maalouf entend faire son examen d'identité , ce n'est point pour retrouver au fond de lui cette appartenance essentielle. C'est tout le contraire, pour rechercher leplus grand nombre d'éléments de son identité.Chacune de ses appartenances le relie à un grand nombre de personnes; plus ces appartenancessont nombreuses, plus son identité s'avèrespécifique.

Cependant, et malheureusement, celui qui aligne ses multiples appartenance "est immédiatement accusé de vouloir "dissoudre" son identitédans une soupe informe où toutes les couleurs s'effacerait. C'est pourtant l'inverse que je cherche à dire. Non pas que tous les humains sont pareils, mais que chacun est différent. ${ }^{14} \mathrm{Il}$ est vrai qu'unchrétien libanais est différent d'un musulman libanais, mais deux chrétiens libanais ne sont certainement pas identiques. "Par facilité, nous englobons les gens les plus différents sous le même vocable, par facilité aussi nous leur attribuons des crimes, des actes collectifs, des opinions collectives- [...], et cela se termine quelquefois dans le sang. ${ }^{15} \mathrm{C}$ 'est bien notre regard qui tend à enfermer les autres dans leurs plus étroites appartenances et c'est notre regard qui peut les libérer.

En effet, le vrai problème commence lorsqu'on incite nos contemporains à retrouver au fond d'eux-mêmes cette «prétendue appartenance fondamentale ${ }^{16}$ religieuse, nationale ou ethnique. On les incite à la «brandir fièrementàla face des autres. ${ }^{17}$ Puis quiconque revendique une identité plus complexe se retrouve marginalisé.

Les exemples abondent et Maalouf ne cite que quelques-uns : un jeune homme né en France de parents algériens, un Turc né il y a trente ans près de Francfort, une personne néeàBelgrade d'une mère serbe et d'un père croate, une femme hutu mariéeà un tutsi.

\footnotetext{
${ }^{13}$ Ibid., p. 17.

${ }^{14}$ Ibid., p.28.

${ }^{15}$ Ibid., p.29.

${ }^{16} /$ bid., p.9.

${ }^{17}$ Ibid., Loc.cit.
} 


\section{هجلة كلية الآتامب. باهعة الإسكندرية ـ المجلد 79 ـ العقد 90.}

Ces êtres qui portent en eux des appartenances qui aujourd'hui s'affrontent violemment sont ce que Maalouf appelle des «êtres frontaliers ». Frontaliers de naissance ou par le hasard de leur trajectoire, ils se trouvent, malgré eux, "traversés par des lignes de fracture ethniques, religieuses ou autres. ${ }^{18}$ Ils ont un rôle majeur à jouer. Ils peuvent tisser des liens. Ils ont pour vocation d'être des traits d'union et des médiateurs entre les diverses communautés, les diverses cultures.Mais ceci est uniquement s'ils assument leur pluralité identitaire. Ceux, par contre, qui ne l'assument paspeuvent devenir parmi les plus virulents des tueurs identitaires.C'est ainsi que Maalouf arrive à décrire avec une finesse extrême et une clairvoyance surprenante comment l'identité devient meurtrière.

Il est vrai qu'après chaque nouveau massacre nous nous demandons, à juste titre d'ailleurs, comment est-ce que des êtres humains en arrivent à commettre de telles atrocités. Nous pouvons parler de folie car, en un sens, il y a bien folie mais cette folie est bien ce que Maalouf explique avec cette grande finesse, cette disposition des hommes à devenir massacreurs une fois leur «tribu » devient menacée.

Or c'est bien lorsque l'identité estconçue de manière «tribale » qu'elle devient meurtrière en opposant «nous» aux «autres ». C'est cette attitude partiale etintolérante qui est à l'origine de tout déchainement identitaire. Cette vision qui réduit l'identité entière et complexeà une seule appartenanceproclamée avec rage: "C'est ainsi que l'on 'fabrique" des massacreurs, ai-je envie de crier! ${ }^{19}$ affirme Maalouf.

La vérité est que si Maalouf insiste sur le fait que l'identité est faite de multiples appartenances, il affirme en même temps qu'elle est «une » et que «ous la vivons comme un tout. ${ }^{20}$ Cette identité n'est point une «juxtaposition d'appartenances autonomes, ce n'est pas un 'patch-work", c'est un dessin sur une peau tendue; qu'une seule appartenance soit touchée, et c'est toute la personne qui vibre. ${ }^{21}$ Et c'est ce moment précis que définit Maalouf qui transforme l'identité blessée en identité meurtrière.

\footnotetext{
${ }^{18}$ Ibid., p.11.

${ }^{19}$ Ibid., p.11.

${ }^{20}$ Ibid., p.34.

${ }^{21}$ Ibid., Loc.cit.
} 


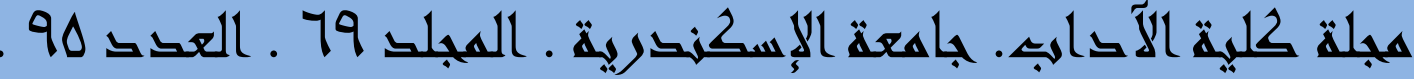

Il est vrai que là où les gens se sentent menacés dans leur foi,c'est l'appartenance religieuse qui semble résumer leur identitéentière. Mais si c'est leur langue maternelle qui est menacée, alors ils se battent farouchement contre leurs propres coreligionnaires. C'est l'appartenance en cause qui envahit toute l'identité. Ceux qui la partagent se sentent solidaires et ils se rassemblent. "Pour eux, "affirmer leur identité" devient forcément un acte de courage, un acte libérateur... ${ }^{22}$

Puis au sein de chaque communautéblesséeapparaissent alors les «meneurs ».

«Enragés ou calculateurs, ils tiennent les propos jusqu'au-boutistes qui mettent du baume sur les blessures.Ils disent qu'il ne faut pas mendier auprès des autres le respect, qui est un dî., mais qu'il faut leleur imposer. Ils promettent victoire ou vengeance, enflamment les esprits, [...]. Désormais, le décor est planté, la guerre peut commencer. Quoi qu'il arrive, "les autres" l'auront mérité, "nous", avons un souvenir précis de "tout ce qu'ils nous ont fait endurer" depuis l'aube des temps. Tous les crimes, toutes les exactions, toutes les humiliations, toutes les frayeurs, des noms, des dates, des chiffres. $»^{23}$

Maalouf est bien conscient, comme nous le sommes tous d'ailleurs, qu'aucune appartenance religieuse, nationale ou autre ne prédispose au meurtre. Ce n'est que lorsqu'une communauté se sente humiliée ou blessée qu'elle aura tendance à produire des tueurs. Maalouf en arrive à comprendre parfaitement pourquoi les massacreurs ont souvent bonne conscience, et s'étonnent de s'appeler criminels.En effet, ces criminels ont toujours l'impression d'agir pour la survie des siens. C'est le cas de tous ceux qui, du Rwanda à l'ancienne Yougoslavie, ont commis les crimes les plus abominables.Ces tueurs, tout en commettantles pires crimes restent «convaincus d'être dans leur droit, de mériter le Ciel et l'admiration de leurs proches. ${ }^{24}$

\footnotetext{
${ }^{22}$ lbid., p.35.

${ }^{23}$ Ibid.,Loc.cit.

${ }^{24}$ Ibid., p.37.
} 


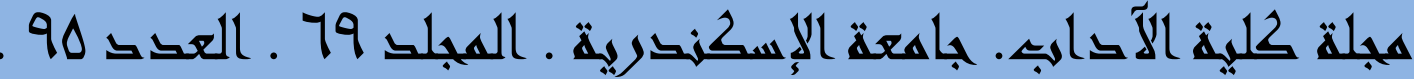

Or cette conception tribale de l'identité est malheureusement celle qui prévaut en ce moment dans le monde entier. Et c'est elle qui favorise la dérive. Cette conception que «beaucoup d'entre nous rejetteraient s'ils l'examinaient de plus près, mais à laquelle nous continuons àadhérer par habitude, par manque d'imagination, ou par résignation, contribuant ainsi, sans le vouloir, aux drames par lesquels nous serons demain sincèrement bouleversés. ${ }^{25}$

C'est en réduisant l'identitéà une seule appartenance qu'on installe les hommesdans une attitudeintolérante suicidaire. Par contre, au moment où on commencera à concevoir notre identité comme étant faite d'appartenances multiples, un rapport différent se crée avec l'autre. Il n'aura plus cette dichotomie simpliste entre «nous » et « eux ». Il y a aura «de "notre" côté , des personnes avec lesquelles je n'ai finalement que très peu de choses en commun, et il y a, de 'leur' côté, des personnes dont je peux me sentir extrêmement proche. ${ }^{26}$

Une des graves conséquences de ces conflits c'est que certains crimes commencentà sembler «compréhensibles et mêmeinévitables ${ }^{27}$. Une «attitude de laisser-tuer ${ }^{28}$ commence àprévaloir. Maalouf dénonce, pour sa part,cette attitude en affirmant que le réalisme dont elle se réclame est usurpé. En effet, si la conception «tribale» de l'identité est celle qui prévaut actuellement dans le monde entier, ceci est malheureusement vrai. Cependant, de nombreuses conceptions qui avaient prévalu pour des siècles, ne sont plus acceptables aujourd'hui. Il suffit de penser à l'esclavage pour comprendre que les idéesqui ont prévalu ne doiventnécessairementprévaloir dans les décenniesà venir. De nouvelles idées peuvent peu à peu s'installer. A nous donc d'y travailler afin de pouvoir un jour changer la réalité des choses.

Sans doute les propos de Maalouf sont-ils ceux d'un migrant, et d'un minoritaire et c'est pourquoi son analyse du problème identitaire le mène inévitablement au deuxième axe de sa pensée : le problème de l'immigration. Il est vrai que le thème de l'immigration lui est familier à titre

\footnotetext{
${ }^{25}$ Ibid., p.38.

${ }^{26}$ Ibid., p.40.

${ }^{27}$ Ibid., p.43.

${ }^{28}$ Ibid., Loc.cit.
} 


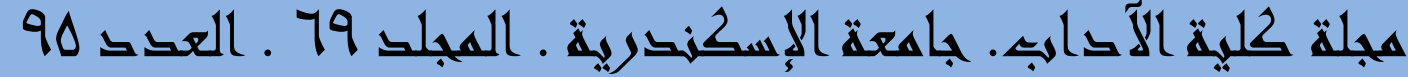

personnel. Maalouf n'hésite pas à partager son expérience personnelle et sa capacité de s'intégrer dans son pays d'accueil sans pour autant renier sa propre culture ni les autres cultures qui l'ont formé.

Cependant, cette situation ne semble point concerner uniquement les écrivains de l'exil.La question de la migration touche un grand nombre d'humains. N'est-ce pas le propre de notre époque que d'avoir fait de tous les hommes, en quelque sorte, des migrants et des minoritaires ? Nous sommes tous contraints de vivre dans un univers qui ne ressemble guèreà notre terroir d'origine; nous devons tous apprendre d'autres langues, d'autres langages et nous avons tous l'impression que notre identité telle que nous l'imaginons depuis l'enfance, est menacée.

Plus de 120 millions de personnes dans le monde ont quitté leurs pays d'origine, dont l'auteur lui-même, pour s'installer dans d'autres pays plus stables, plus libres et plus prospères que les leurs. La présence massive d'immigrés musulmans dans les grandes villes d'Europe crée de plus en plus une atmosphère de méfiance et de répulsion. Ces immigrés, appréciés au temps des années prospères de l'Europe d'après-guerre, sont devenues de trop, surtout après la récession économique qui a frappé de nombreux pays européens.

Mais aussi et surtout, c'est dans le contexte de l'immigration que les problèmes identitairespeuvent devenir meurtriers. Le migrant se trouve,malgré lui, dans l'obligation de choisir une seule appartenance qui compte. S'il lui faut choisir, il se trouve obligéde trahir soit sa patrie, soit son pays d'accueil. Ce déchirement s'accompagne d'un sentiment de culpabilité car "il y a des proches que l'on s'en veut d'avoir abandonnés, une maison où l'on a grandi, tant et tant de souvenirs agréables.» ${ }^{29}$ Cependant, si on est parti, c'est qu'il y a des choses que l'ona rejetées - la répression, l'insécurité ou la pauvreté.

Puis, dans le pays d'accueil, on affronte d'autres sentiments non moins ambigus. "Le premier réflexe n'est pas d'afficher sa différence, mais de passer inaperçu. ${ }^{30}$ Certains choisissent d'imiter leurs hôtes.Quelquefois, ils y parviennent mais le plus souvent, ils n'y parviennent

\footnotetext{
${ }^{29}$ Ibid., p. 48.

${ }^{30}$ Ibid., Loc.cit.
} 


\section{هجلة كلية الآتامب. باهعة الإسكندرية ـ المجلد 79 ـ العقد 90.}

pas. Beaucoup, sachant que ce n'est même pas la peine d'essayer,se montrent par fierté oupar bravade, plus différents qu'ils ne le sont.

Les deux conceptions paraissent pour Maalouf «irréalistes, stériles et nuisibles. ${ }^{31}$ La solution est bien au juste milieu entre les deux. Le pays d'accueil n'est point un terrain vague où chacun pourrait s'installer, sans rien changer à ses habitudes. Ce n'est pas non plus une terre dont les lois, les valeurs, les croyances, les caractéristiques culturelles et humaines auraientdéjàétéfixées une fois pour toutes, les immigrants n'ayant plus qu'à s'y confirmer.«[...] le pays d'accueil n'est ni une page blanche, ni une page achevée, c'est une page en train de s'écrire. ${ }^{32}$

En fait, tout l'avenir de l'Europe dépend de cette volonté d'intégrer les nouveaux arrivés et de leur permettre de prendre part à la vie quotidienne. Si l'effort ne se fait pas des deux côtés, la tentative aboutira à un échec fatal.

Tout en admettant quel'histoire doit êtrerespectée, l'avenir ne peut être un simple prolongement de l'histoire. Il faudra de profondes transformations.

"[...] j'aurais envie de dire, 'aux uns"' d'abord: 'Plus vous vous imprégnerez de la culture du pays d'accueil, plus vous pourrez l'imprégner de la vôtre"'; puis "aux autres": "Plus un immigré sentira sa culture d'origine respectée, plus il s'ouvrira à la culture du pays d'accueil. » » ${ }^{33}$

Ces deux « équations », se tiennent inséparables selon Maalouf, « comme les pieds d'un escabeau. ${ }^{34}$

Ainsi, le maître mot pour Maalouf pour résoudre les problèmes des immigrants est-il la réciprocité. Il invite les uns et les autres à faire de la réciprocité leur souci primordial, seule garante d'un pacte social durable, dans les pays où il y a une forte concentration d'immigrés. C'est d'abord à l'immigré de faire l'effort nécessaire mais c'est également au pays de faire

\footnotetext{
${ }^{31}$ lbid., p. 50.

${ }^{32}$ Ibid., Loc.cit.

${ }^{33}$ Ibid., p.51.

${ }^{34}$ Ibid., Loc.cit.
} 


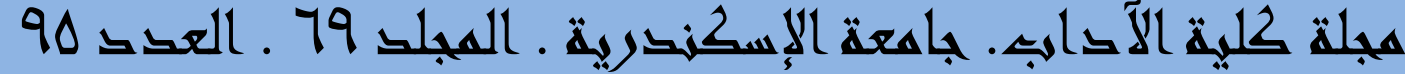

cet effort d'intégration. C'est cette réciprocité qui permettrait de réduire les écarts car si le fossé entre autochtones et immigrés se creuserait davantage, il favorisera le communautarisme et le brandissement aveugle de l'identité.

Lorsqu'on sent sa langue méprisée, sa religion bafouée, sa culture dévalorisée, on réagit en affichant avec ostentation les signes de sa différence. Par contre, lorsqu'on se sent respecté, lorsqu'on sent sa place dans le pays où l'on a choisi de vivre, alors on réagit autrement.

Ainsi, l'exil se trouve-t-il au centre de tous les romans de Maalouf dans une claire opposition entre l'intérieur et l'extérieur, l'appartenance et l'exclusion. Les Désorientés, son dernier roman est un roman de l'exil et du retour. L'orient y apparait comme une terre hostile qui a transformé ses citoyens en exilés, y compris ceux qui sont restés.

Le roman dresse le tableau des désillusions d'une génération. Celle de ces hommes et femmes qui avaient 20 ans dans les années 1970 et se réveillent des décennies plus tard, hébétés. Il met en scène une ancienne bande d'amis réunis au début de l'année 2001. Dans le roman s'érige une contradiction entre ceux qui sont partis et ceux qui sont restés, et ce, sur le mode de la trahison. Ceux qui sont partis ont le luxe de pouvoir se dire «purs » puisqu'ils n'ont pas eu à vivre la guerre et tous les compromis, concessions et collaborations qu'elle a pu entrainer. De même, ceux qui sont restés peuvent se targuer d'être restés loyaux en n'abandonnant pas le pays, d'être restés sur place pour le meilleur et pour le pire, sans céder aux promesses d'une vie riche et facile.

Cette douloureuse approche des identitésdéchirées par l'immigration se retrouveégalement dans Le premier siècleaprèsBéatrice. "La ruine des pays du Sud, causée par l'effroyable marasme d'une civilisation sur le point de mourir, entraine un sauve-qui-peut vers les pays du Nord. Les élites, les prévoyants, en somme tous ceux qui peuvent se le permettre, s'empressent de quitter les régions de plus en plus étendues qui implosent petit à petit. » ${ }^{35}$

Dans ce roman de science-fiction, les migrations humaines sont comparées aux migrations animales, notamment celle des uranies, une espèce de papillon qui effectue, chaque année, un suicide collectif sous la forme d'une migration au-dessus de l'océan. L'hypothèse de l'entomologiste, qui se passionne pour cette espèce, est que ce

\footnotetext{
${ }^{35}$ BOUVET, Rachel et EL KETTANI, Soundous, Op.cit., p.154.
} 
comportement provient « de la survivance d'un réflexe ancestral qui conduit encore ces bestioles vers un lieu où elles se reproduisaient autrefois, peutêtre une île qui aurait disparu; ainsi, leur suicide apparent serait un acte involontaire causé par une mauvaise adaptation de l'instinct de survie à des réalités nouvelles. ${ }^{36}$

Ce vol des uranies entraine la réflexion sur les migrations humaines. On ne peut manquer de penser aux migrations illégales des milliers de jeunes qui quittent les côtes nord-africaines avec le rêve d'une vie meilleure en Europe, rêve qui malheureusement finit pour la grande majoritédans les cimetières de Lampedusa.

De tous les conflits identitaires qui opposent les immigrants aux autochtones, Maalouf choisit de se pencherparticulièrement sur le cas des immigrants arabes et musulmans ce qui nous mène au troisième axe de sa pensée: l'islam et la modernité où il cherche à répondre à cette question : "L'islam est-il incompatible avec la liberté, avec la démocratie, avec les droits de l'homme et de la femme, avec la modernité? » ${ }^{37}$

Or, la réponse s'avère d'une grande complexité vu l'état du monde arabe. Des voix de plus en plus nombreuses s'élèvent en Europe, en Amérique du Nord, voire même dans les pays arabo-musulmans, accusant l'islam d'archaïsme, de passéisme et de violence.

Maalouf renvoie dos à dos, ceux qui condamnent systématiquement l'islam et l'accusent des maux du monde et ceux qui considèrent que ces actes exécrables qui se produisent ici et là résultent d'un malentendu. Pour lui, c'est une certaine interprétation de la doctrine musulmane qui est condamnable et même par de nombreux musulmans.

En fait, «le texte ne change pas, c'est notre regard qui change. Mais le texte n'agit sur les réalités du monde que par le biais de notre regard. Lequel s'arrêteà chaque époque sur certaines phrases et glisse sur d'autres. » ${ }^{38}$ Ainsi, ce n'est pas sur l'essence de la doctrine qu'il faut se pencher mais sur le comportement de ceux qui s'en réclament.

Maalouf rappelle comment tout au long de son histoire, le christianisme a connu des revers, non point en raison de son essence mais en

\footnotetext{
${ }^{36}$ Le premier siècle après Béatrice, Paris, Grasset, 1992, p.155

${ }^{37}$ Les Identités meurtrières, p. 57

${ }^{38}$ Ibid., p.59.
} 


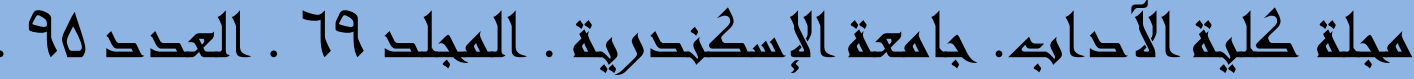

raison d'une certaine interprétation au profit des autorités ecclésiastiques et politiques à la fois. On a torturé, persécuté et massacré abondamment au nom de la religion. "Les plus hautes autoritésecclésiastiques ainsi que l'écrasantemajorité des croyants se sont accommodées de la traite des Noirs, de l'assujettissement des femmes, des pires dictatures, comme de l'Inquisition. $\gg{ }^{39} \mathrm{Ceci}$ ne veut point dire que le christianisme est par essence intolérant. Il suffit de regarder autour de soi pour constater comment il défend aujourd'hui les droits de l'homme et la démocratie.

Il est vrai que le XXe siècle nous a appris qu' «aucune doctrine n'est, par elle-même, nécessairementlibératrice, toutes peuvent déraper, toutes peuvent être perverties, toutes ont du sang sur les mains, le communisme, le libéralisme, le nationalisme, chacune des grandes religions, et même la laïcité. Personne n'a le monopole du fanatisme et personne n'a, à l'inverse, le monopole de l'humain. » ${ }^{40}$

Encore une fois, la vision de Maalouf opère ce déplacement de perspective significatif. En retournant à l'histoire, il noteque l'islam s'est traditionnellement accommodé de la présence, sur les terres qu'il contrôlaitdes adeptes des autres religions monothéistes.Par contre, "Que sont devenus, [...], les musulmans d'Espagne? Et les musulmans de Sicile? Disparus, tous jusqu'au dernier, massacrés, contrains à l'exil ou baptisés de force. ${ }^{41}$

En effet, il y a dans l'histoire de l'islam, dès ses débuts, une remarquable capacitéà coexister avec l'autre.Rappelons qu'à la fin du siècle dernier, Istanbul, capitale de la principale puissance musulmane, comptait dans sa population une majorité de non-musulmans, principalement des Grecs, des Arméniens et des Juifs.

Cette pratique de la coexistence dans l'histoire musulmane provient $\mathrm{du}$ fait que "L'islam avait établi un "protocole de tolérance" à une époqueoù les sociétéschrétiennes ne toléraient rien. Pendant des siècles, ce "protocole" fut, dans le monde entier, la forme la plus avancée de coexistence. $\gg{ }^{42}$

\footnotetext{
${ }^{39}$ Ibid., p.60.

${ }^{40}$ Ibid., p.62.

${ }^{41}$ lbid., p.67.

${ }^{42}$ Ibid., p.68.
} 


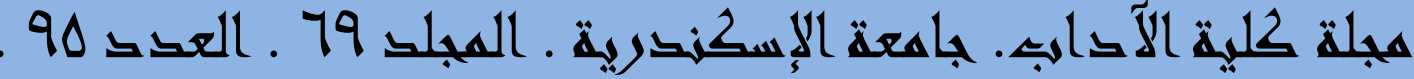

Ceci dit, en vantant la tolérance du passé, Maalouf ne cherche aucunement à dissimuler les atrocités du présent. La vérité est quece «protocole de tolérance» n'a pas été remis à jour. Si bien que le monde musulman, après avoir été, pendant des siècles, à la pointe de la tolérance, s'est retrouvéà la traine.

Si l'histoire montre que l'islam porte en lui d'immenses potentialités de coexistence avec les autres cultures, l'histoire plus récente montre qu'une régression est possible. En faisant l'histoire comparée du monde chrétien et du monde musulman, on trouverait d'un côté une religion longtempsintolérante, mais qui s'est peu à peu muée en une religion d'ouverture, et de l'autre côté une religion porteuse d'une vocation d'ouverture, mais qui a peuà peu dérivé vers des comportementsintolérants et totalitaires.

Mais comment expliquer cette transformation ? Pour Maalouf, on a beau parlé de l'influence des religions sur les peuples alors qu'il ne fait point ignorer l'influence des peuples sur les religions. " [...] si le christianisme a façonné l'Europe, l'Europe aussi a façonné le christianisme. Le christianisme est aujourd'hui ce que les sociétéseuropéennes en ont fait. » ${ }^{43}$

Or, l'islam comme toute autre doctrine porte la marque de son temps. L'hostilité des musulmans aux idées de la modernité n'est en fait qu'un manque de confiance. Maalouf interpelle l'histoire pour prouver que les musulmans, lorsqu'ils étaient confiants et lorsqu'ils triomphaient, ils pratiquaient l'ouverture sans aucun complexe et interprétaient leur foi dans un esprit de tolérance et d'ouverture.

«Les sociétés sures d'elles se reflètent dans une religion confiante, sereine, ouverte; les sociétés mal assurées se reflètent dans une religion frileuse, bigote, sourcilleuse. Les sociétés dynamiques se reflètent en un islam dynamique innovant, créatif; les sociétés immobiles, se reflètent en un islam immobile, rebelle au moindre changement. » ${ }^{44}$

C'est ainsi que l'observation de l'histoire mène Maalouf à comprendre que les mouvements islamistes militants d'aujourd'hui ne sont

\footnotetext{
${ }^{43}$ lbid., p.72.

${ }^{44}$ Ibid. p.p.75, 76
} 


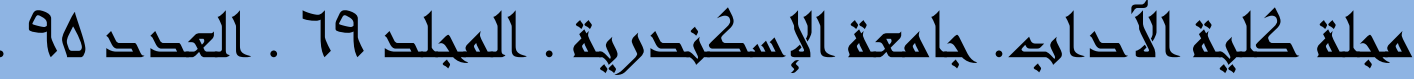

pas un pur produit de l'histoire musulmane, ils sont le produit de notre époque.

Maalouf refuse l'habitude qui classe chaque événement se déroulant dans chaque pays musulman sous la rubrique 'islam', alors que bien d'autres facteurs entrent en jeu.

En fait, ceux qui rendent l'islam responsable de tous les drames, rendent en effet les événements du monde totalement inintelligibles. On a d'ailleurs dit du christianisme la même chose pendant des siècles avant de découvrir qu'il était capable de se moderniser.

Selon Maalouf, il faudra « encore du temps, beaucoup de temps, [...], avant qu'on puisse avoir la preuve que ce spectacle qui s'offre à nous, en Algérie, en Afghanistan, un peu partout, fait de violence, d'archaïsme, de despotisme, de répression, n'est pas plus inhérentà l'islam que les buchers des inquisiteurs ou la monarchie de droit divin ne se sont avérésinséparables du christianisme. ${ }^{45}$

Samuel Huntington avait bien vu cette fracture au début des années 90. L'auteur du Choc des civilisations considérait que le conflit identitaire le plus marquant depuis la fin de la guerre froide serait sans doute entre l'Occident et le monde musulman.

Encore une fois, Maalouf remonte dans l'histoire pour retrouver l'origine du clivage entre le Nord et le Sud car ce renversement du rapport de forces est assez récent. Entre le XV et le XIX e siècle, alors que l'Occident avançaittrès vite, le monde arabe piétinait. Depuis la Renaissance, un vent de pensée libre s'est emparé de l'Europe, en particulier, de l'art italien, et par la suite, de la littérature, de la philosophie et de la science. Ce changement extraordinaire a progressivement marqué la découpe historique entre le Moyen-Âge et l'âge moderne et a mis l'Europe sur une voie de modernisation ininterrompue.

Cet esprit libre a permis à l'Europe de se lancer dans une aventure culturelle inégalable. Il a gagné non seulement les intellectuels mais aussi le peuple. C'est cet esprit qui a pu moderniser sa religion. Par contre, dans le monde musulman, les choses ne se sont pas passées de la même

\footnotetext{
${ }^{45}$ Ibid., p. 80

${ }^{46}$ HUNTINGTON, Samuel, Le Choc des civilisations, Paris, Odile Jacob, 2000, p.312.
} 


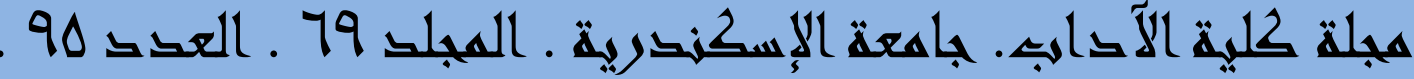

manière.« Pas parce que cette religion-là n'était pas 'modernisable"'- [...], mais parce que la société elle-même ne s'est pas modernisée. » ${ }^{47}$

Mais pourquoi en Occident, et pas en Chine ou pas au Japon, pas en Russie, ou même pas dans le monde arabe ? Ce qui est certain c'est qu'aujourd'hui, l'Occident est partout. Tout ce qui influence les hommes vient de l'Occident. "Où que l'on vive sur cette planète, toute modernisation est désormais occidentalisation. ${ }^{48}$

Un peu partout, il y a, certes, des monuments et des ouvrages qui portent l'empreinte de civilisations spécifiques. Mais tout ce qui se crée de neuf vient de de l'Occident.

Or, cette réalité n'est pas vécue de la mêmemanière par ceux qui sont nés au sein de la civilisation dominante et par ceux qui sont nés en dehors. En effet, les occidentauxpeuvent avancer dans la vie sans cesser d'êtreeux-mêmes. On pourrait même dire que, "pour les Occidentaux, plus ils se modernisent, plus ils se sentent en harmonie avec leur culture, seuls ceux qui refusent la modernité se retrouvent déphasés. ${ }^{49}$

Pour le reste du monde, la situation est différente car la modernisation implique l'abandon d'une partie de soi-même. Elle ne se déroule jamais sansune profonde crise d'identité.

Ainsi, lorsqu'unesociété voit dans la modernité ''la main de l'étranger', elle a tendance à la repousser et à s'en éloigner. "Quand la modernité porte la marque de 'l'Autre', il n'est pas surprenant de voir certaines personnes brandir les symboles de l'archaïsme pour affirmer leur différence. $\gg{ }^{50}$ Cette réaction de rejet systémique va jusqu'àêtre suicidaire.

Ceci est même le cas en France, où la mondialisation apparaît aujourd'hui comme synonyme d'américanisation. Les Français refusent lefast food, Hollywood, CNN, Disney et Microsoft.

Encore une fois, c'est ce déplacement de perspective qui permetàMaalouf d'imaginer le sentiment qu'éprouvent les différents peuples non-occidentaux pour qui chaque pas s'accompagne d'un sentiment de négationde soi. Il leur faut admettre que leur savoir est dépassé, que leur

\footnotetext{
${ }^{47}$ Les Identités meurtrières, p.80

${ }^{48}$ Ibid., p.p.83, 84.

${ }^{49}$ Ibid., p.84.

${ }^{50}$ Ibid., p. 85
} 


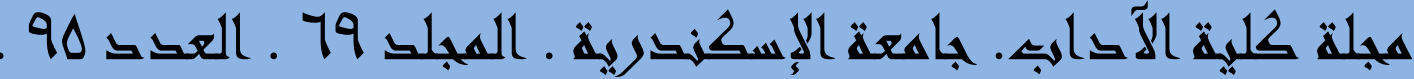

médecine traditionnelle n'est que superstition, que leurs grands hommes ne comptaient pour rien pour le reste du monde, que leur religion est suspecte de barbarie, qu'ils devaient étudier d'autres langues pour garder le contact avec le reste du monde. Lorsqu'ils parlent avec un Occidental, c'est toujours dans sa langue à lui, jamais dans la leur.

Les Arabes recherchent dans les siècles lointains des raisons d'être fiers. Ils ont besoin d'invoquer l'algèbre, l'algorithme et le zéro pour se vanter. Par contre, les Japonais et les Chinois ont su se faire respecter dans le monde entier bien qu'ils restent attachés à leurs traditions et leurs cultures. La vérité c'est que les Arabes n'ont pas réussi à entrer dans la modernité et à profiter de ses réalisations comme le Chinois, les Japonais ou les Coréens. Ils l'ont même repoussée parce qu'elle vient précisément de l'Occident.

La vraie solution à ce problème de refus de la modernité car elle vient de chez l'autre, est que dans la civilisation globale, telle qu'elle est en train de se construire, ne soit pas exclusivementaméricaine. Il faut que chacun puisse s'y reconnaître un peu, que chacun puisse s'identifier un peu à elle, que personne ne soit amenéàconsidérer qu'elle lui est inévitablementétrangère, et de ce fait hostile.Maalouf touche ainsi au fond du problème dans le rapport entre un Occident triomphaliste et un monde arabo-musulman frustré qui affiche violemment son identité religieuse pour défendre sa propre identité.

Cette «montée du phénomène religieux ${ }^{51}$ surprend car malgré la complexité de l'identité de chacun et ses multiples appartenances, àl'heure actuelle, affirmer son appartenance religieuse, la considérer comme l'élément central de son identité, est une attitude courante «moins répandue, sans doute qu'il y a trois cents ans, mais indiscutablement plus moins répandue qu'il y a cinquante ans. » ${ }^{52}$

Ces mêmes personnes qui se sentent poussées à affirmer leur appartenance religieuse, quelques années plus tôt, auraient choisi de mettre en avant, spontanément, d'autres appartenances.

Maalouf remonte dans l'histoire pour retrouver l'origine de ce phénomène. Le déchaînement identitaire qui s'intensifie dans le monde arabo-musulman n'est en fait qu'une conséquence de l'histoire brisée :

\footnotetext{
${ }^{51}$ Ibid., p.100.

${ }^{52}$ lbid.,p.99.
} 


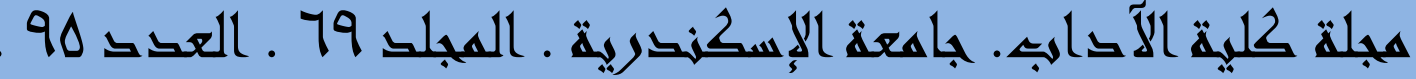

«Il a fallu que les dirigeants nationalistes, Nasser en tête, arrivent à une impasse, tant par leurs échecs militaires successifs que par leur incapacité à résoudre les problèmes liés au sous-développement, pour qu'une partie significative de la population se mette à prêter l'oreille au discours du radicalisme religieux, et pour qu'on voie fleurir, à partir des années 1970, voiles et barbes protestataires.[...] Je voulais seulement redire ici, encore et encore, que le radicalisme religieux n'a pas été le choix spontané, le choix naturel, le choix immédiat des Arabes ou des Musulmans. Avant qu'ils ne soient tentés par cette voie, il a fallu que toutes les autres se bouchent. ${ }^{53}$

Maalouf rappelle également que l'effondrement du communisme avait réhabilitéles croyances qu'il avait voulu rejeter.Après la guerre froide, l'influence des islamistes était devenue visible sur la vie quotidienne. Il importe de rappeler dans ce contexte que les islamistes s'étaient coalisés avec les Américains pour mettre fin à l'occupation de l'Afghanistan par les soviétiques, ennemi commun des deux. Une fois l'objectif atteint, les rapports avec l'Occident se sont détériorés et n'ont cessé de se compliquer depuis.

D'autre part, Maalouf rappelle également que «le nationalisme arabe [qui a été] confisqué par des régimes autoritaires, incompétents et corrompus, a perdu de sa crédibilité. » ${ }^{54}$

L'Occident apparaît donc comme le paradis. Tous ceux qui le pouvaient ont choisi d'émigrer. Tous ceux qui n'ont pas pu ou voulu émigrer se sont trouvés tentés par la mouvance islamiste qui leur a permis d'assouvir à la fois un besoin d'identité et un acte de révolte.

Maalouf pousse encore plus loin son analyse pour trouver dans l'affirmation de l'appartenance religieuse «une initiative de synthèse entre le besoin d'identité et l'exigence d'universalité. » ${ }^{55}$ Les communautés de croyants paraissent, selon lui,comme des «tribus planétaires», «je dis "tribus" à cause de leur teneur identitaire, mais je dis aussi "planétaires", parce qu'elles enjambent allègrement les frontières. L'adhésionà une foi qui

\footnotetext{
${ }^{53}$ Ibid., p.96.

${ }^{54}$ Ibid., p.p.101, 102.

${ }^{55}$ Ibid., p.106.
} 


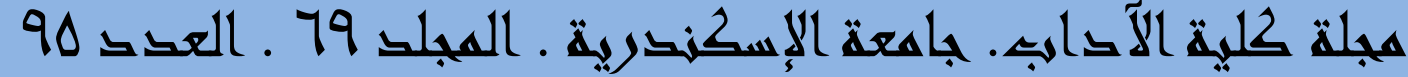

transcenderait les appartenancesnationales, raciales, sociales, apparait aux yeux de certains comme leur manièreà eux de se montrer universels. » ${ }^{56}$

Mais la vraie question que pose Maalouf est bien: «vers quoi va-t-on dépasser l'appartenance religieuse? Quelle autre appartenance va pouvoir la rendre "obsolète" comme elle sembla l'êtrenaguère? " ${ }^{57} \mathrm{Tel}$ que le formule le héros dans Les Désorientés, "Hier comme aujourd'hui, Dieu est un refuge pour les vaincus, leur ultime secours. Au nom de quoi voudrais-tu les en priver? Et pour le remplacer par quoi? $\gg{ }^{58}$

Il est vrai quedépasser l'appartenance religieuse ne veut point dire dépasser la religion elle-même. Maalouf ne rêvepoint d'un monde sans religion, il rêve "d'un monde où la religion ne servirait plus de ciment à des ethnies en guerre. ${ }^{59} \mathrm{C}^{\prime}$ est dans ce sens qu'il entend séparer le religieux de l'identitaire et satisfaire autrement le besoin d'identité.

Cependant, l'appartenance religieuse ne pourrait pas êtresupplantée par des appartenances traditionnelles comme la nation, l'ethnie ou la race. Toutes s'avèrentétroites et limitatives, du coup non moins meurtrières. S'il faut dépasser l'appartenance religieuse, c'est vers une appartenance plus vaste et plus générale.

La seule et vraie solution revient donc encore une foisàla pluralité de l'identité. La solution est que l'identité soitperçue comme la somme de toutes nos appartenances. Seule, "l'appartenance à la communauté humaine prendrait de plus en plus d'importance, jusqu'à devenir un jour l'appartenance principale, sans pour autant effacer nos multiples appartenances particulières - je n'irai sûrement pas jusqu'à dire que le "vent'" de la globalisation nous pousse obligatoirement dans cette direction mais il me semble qu'il rend une telle approche moins difficileà envisager. Et dans le même temps, indispensable. " ${ }^{60} \mathrm{Par}-\mathrm{là}$, on arrive au dernier axe de la pensée de Maalouf : celui de la mondialisation.

Pour répondreà la question de la mondialisation, si elle est salutaire ou maléfique, Maalouf dénonce deux tendances extrêmes, à savoir l'affirmation démesurée del'identité ou,à l'inverse, la dissolutionde celle-ci, autrement dit,

\footnotetext{
${ }^{56}$ Ibid., Loc.cit.

${ }^{57}$ Ibid., p.107.

${ }^{58}$ Les Désorientés, Paris, Grasset, 2012, p.502.

${ }^{59}$ Les Identités meurtrières, p.110.

${ }^{60}$ Ibid., p.115.
} 


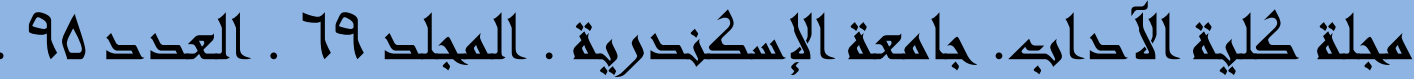

le fanatisme ou la perte de tout repère. Il propose quant à lui, une troisième voie, plus subtile, plus efficace qui consiste à établir un équilibre harmonieux entre l'appartenance légitime à une identité et l'ouverture intelligente à la mondialisation.

La vérité est que, sous l'effet de la mondialisation culturelle, nous devenons de moins en moins différents et c'est peut-être pourquoi nous affirmons violemment nos différences.

Si la mondialisation apparaît comme un formidable brassage, elle risque d'être une uniformisation appauvrissante, et une menace contre laquelle il faut se battre pour préserver sa propre culture, son identité et ses valeurs. Tel que le formule Maalouf, «la mondialisation nous entraine, d'un même mouvement, vers ces deux réalitésopposées: "l'universalité et l'uniformité. » ${ }^{61}$

Toutes les particularités et les spécificités que les sociétés humaines ont érigé au fil des siècles pour marquer leur différences et renfoncer leurs frontières subissent une pression sans précèdent pour réduire ces différences et supprimer ces frontières.

Maalouf n'est point pour un monde monocorde et puéril. Tout au contraire, il lutte contre l'uniformisation appauvrissante, contre l'hégémonie qu'elle soit idéologique, politique, économique ou médiatique. Le combat pour la défense des traditions culturelles est, selon lui, un combat primordial.

D'autre part, le projet de Maalouf pour la diversité culturelle porte aussi sur la diversité linguistique. Parmi les différentes appartenances, Maalouf relève que si la religion a vocation à être exclusive, la langue ne l'est point. " [...] la langue a cette merveilleuse particularité d'être à la fois facteur d'identité et instrument de communication. Pour cela, et contrairement au souhait que je formulais s'agissant de la religion, séparer le linguistique de l'identitaire ne me paraît ni envisageable, ni bénéfique. La langue a vocation à demeurer le pivot de l'identité culturelle, et la diversité linguistique le pivot de toute diversité. » ${ }^{62}$

Si la langue identitaire assure la cohésion nationale, elle risque d'être elle aussi «meurtrière ».La connaissance de la langue de l'autre est la première étape pour favoriser l'échange.

\footnotetext{
${ }^{61}$ lbid., p.121.

${ }^{62}$ lbid., p.p.153, 154.
} 


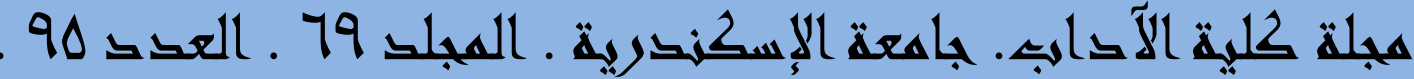

Or la situation aujourd'hui est compliquée. Pour qu'une personne puisse se sentir à l'aise dans le monde d'aujourd'hui, elle doit abandonner sa langue identitaire. Elle doit " 's'expatrier" mentalement" ${ }^{63}$ chaque fois qu'elle doit lire ou même réfléchir. L'anglais devient la langue obligatoire de communication avec le monde.

Maalouf trouve la solution à cette situation dans le trilinguisme. Il affirme que dans le monde d'aujourd'hui, chacun a besoin de trois langues. La première est la langue identitaire qu'il faut préserver. La troisième est l'anglais pour pouvoir s'ouvrir sur le monde. Mais entre les deux, Maalouf affirme qu'il faut obligatoirement promouvoir une deuxième langue, librement choisie, "qui serait souvent, mais pas toujours, une langue européenne. [...], la langue de cour, la langue adoptive, la langue épousée, la langue aimée ... » ${ }^{64}$

C'est justement cette deuxième langue qui assurera les courants d'échanges entre les cultures car «Demain, les relations entre l'Allemagne et la France, seront-elles entre les mains des anglophones des deux pays, ou bien entre les mains des Allemands francophones et des Français germanophones? $\gg 5$

Ce sera effectivement un sérieux handicap de ne pas connaître l'anglais, mais ce sera aussi, et de plus en plus, un sérieux handicap de ne connaître que l'anglais. S'il faut généraliser, l'enseignement de l'anglais comme troisième langue, il faut expliquer "inlassablement aux jeunes à quel point il est à la fois nécessaire et insuffisant ; [...]. » ${ }^{66}$

Telle semblerait être la voie de la sagesse pour pouvoir tirer du formidable essor des communications l'enrichissement, à tous les niveaux, plutôt que l'appauvrissementet la méfiance généralisée.

Selon Maalouf, seule la «réciprocité » pourrait mener à une interaction généreuse et bienveillante entre les cultures. Or, cette réciprocité ne devient bénéfique que si chacun puisse sentir que certains éléments de sa culture font partie du patrimoine universel. Ainsi, l'affirmation de la

\footnotetext{
${ }^{63}$ Ibid., p.159

${ }^{64}$ Ibid., p.162.

${ }^{65}$ Ibid., p.p.162, 163.

${ }^{66}$ Ibid., p.163
} 


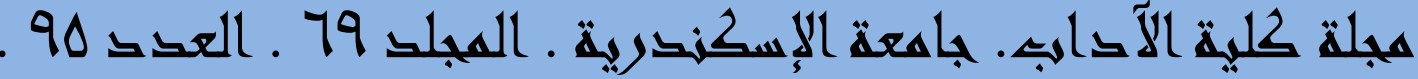

pluralitéidentitaire ${ }^{67}$ apparaît-elle comme la seule vraie solution à tous ces problèmes.

Les convictions humanistes de Maalouf le conduisentàcette définition ouverte del'identité : ouverte sur soi d'abord; ouverte ensuite sur les autres. L'universel chez lui est «une aspiration, une idéegénéreuse, un idéal en perpétuelle construction. $"{ }^{68}$ Cet universel apparaît comme l'unique façon où les divers apports des différents espaces convergent, s'intègrent et se fondent de manière harmonieuse. Son postulat rejoindrait celui senghorien d' "une civilisation de l'universel », symbiose de toutes les civilisations différentes, réalisée historiquement par osmose et intégration harmonique des identités culturelles. ${ }^{69}$ Cette notion s'oppose, selon Senghor, à celle de civilisation universelle dont le poètesénégalais redoutait les menaces sur la cohésion et l'équilibre de l'humanité. La civilisation de l'universel est proposée donc comme alternative à la mondialisation.

La vraie question que pose Maalouf pour terminer sa réflexionsur la mondialisation est bien «à qui donc appartient le monde ? ${ }^{70}$ Et en effet, sa réponse vient "A aucune race en particulier, à aucune nation en particulier. Il appartient, plus qu'à d'autres moments de l'Histoire, à tous ceux qui veulent s'y tailler une place. ${ }^{71} \mathrm{Si}$ le monde d'aujourd'hui transforme profondément notre vision de nous-même, il donne aussi, à ceux qui veulent préserver les cultures menacées, les moyens de se défendre.

Amin Maalouf ne cherche point à cacher les laideurs du monde. Ses outrances et ses inégalités ne sont que trop visibles. La mondialisationne menace la diversité culturelle que si nous n'y prenons pas garde. Les obstacles qui séparent les peuples les uns des autres, si inextricables soientils, sont surmontables pour Maalouf.

Chaque culture présente des traits originaux qui lui permettent de révéler un esprit unique et irremplaçable. Il faut respecter toute culture, si

\footnotetext{
${ }^{67}$ Cette conception de pluralité identitaire nous renvoie à la notion d'hybridité tel que la définit Homi Bhabha et qui lui permet d'appréhender la pluralité des traits culturels dans Les lieux de la culture. Une théorie postcoloniale, Paris, Payot, 2007.

${ }^{68}$ BOUVET, Rachel et EL KETTANI, Soundous, Op.cit., p.203.

${ }^{69}$ SENGHOR, Léopold Sédar, Liberté III : Négritude et civilisation de l'universel, Paris, Seuil, 1977. Cité par Ibid., p.p.203, 204.

${ }^{70}$ Les Identités meurtrières, p.145.

${ }^{71}$ Ibid., Loc.cit.
} 


\section{هجلة كلية الآتامب. باهعة الإسكندرية ـ المجلد 79 ـ العقد 90.}

minime soit-elle, et la considérer comme faisant partie du patrimoine universel. C'est la seule voie pour consolider la paix et la coexistence dans le monde.

Quant au désir d'identité,selon Maalouf, il «ne doit être traité ni par la persécution ni par la complaisance, mais observé, étudié sereinement, compris, puis dompté, apprivoisé, si l'on veut éviter que le monde ne se transforme en jungle, si l'on veut éviter que l'avenir ne ressemble aux pires images du passé, si l'on veut que dans cinquante ans, dans cent ans, nos fils ne soient encore obligés d'assister, comme nous impuissants aux massacres, aux expulsions, et autres "purifications" - d'y assister, et quelquefois de les subir. $\gg^{72}$

Maaloufrêve d'un jour où chacun pourra être jugé pour ses qualités humaines et ses opinions et non sur ses appartenances. Chacun devra être capable d'assumer sa propre identité et de la concevoir comme la somme de toutes ses appartenances, "au lieu de la confondre avec une seule, érigée en appartenance suprême, et en instrument d'exclusion, parfois en instrument de guerre. ${ }^{73}$ Maalouf rêve d'une société multiculturelle où les identités sont plurielles sans être conflictuelles.

Quant à ceux qui vivent dans une société qui ne coïncide pas avec leur culture d'origine, il faut « qu'ils puissent assumer sans trop de déchirements cette double appartenance, maintenir leur adhésionà leur culture d'origine, ne pas se sentir obligés de la dissimuler comme une maladie honteuse, et s'ouvrir parallèlementà la culture du pays d'accueil. $\gg^{74}$

Bien entendu, un tel horizon ne peut être atteint du jour au lendemain "mais ce n'est pas une raison pour conduire l'attelage dans la direction opposée. ${ }^{75} \mathrm{Sa}$ conviction est que l'avenir n'est écrit nulle part et l'avenir sera ce que nous en ferons.

«Aujourd'hui on sait que l'Histoire ne suit jamais le chemin qu'on lui trace.[...]L'Histoire avance à chaque instant sur une infinité de chemins. [...] L'avenir sera-t-il celui de nos espérances ou bien celui de nos cauchemars? ${ }^{76}$

\footnotetext{
${ }^{72}$ lbid., p.165

${ }^{73}$ Ibid., p.183.

${ }^{74}$ Ibid., Loc.cit.

${ }^{75}$ Ibid., p.p.172, 173.

${ }^{76}$ Ibid., p.112
} 


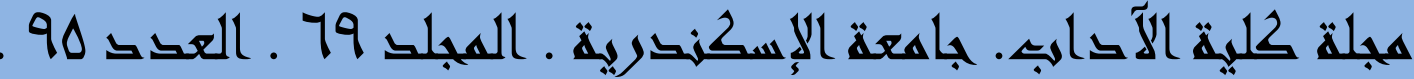

Le "vent' de la mondialisation pourrait effectivement nous conduire au pire, mais également au meilleur. C'est notre choix qui détermine notre l'avenir.

Cet intérêt que porte Maalouf pour l'avenir acquiert parfois un aspect prophétique éblouissant pour le lecteur. «Si nul n'est prophète en son pays, qui mieux qu'Amin Maalouf exerce sa voix prophétique, autrement dit sa clairvoyance? $\gg{ }^{77}$ Depuis Les jardins de Lumières (1991), jusqu'au Identités meurtrières (1998) et aux Désorientés (2012), la prophétie résonne dans toute l'œuvre de Maalouf, le plus souvent à travers le spectre du déséquilibre Nord-Sud tel que le note Caroline Mangurel. ${ }^{78}$ En effet, plusieurs événements de la dernière décennie correspondent justement à ses prévisions, notamment l'emploi de plus en plus répandu, voire banalisé, du terrorisme-suicide.

Dans Les Désorientés (2012), son tout dernier roman, Maalouf prévoit le futur en imaginant le devenir du monde, y entrainant de force le lecteur. Albert, l'un des personnages principaux,scientifique exilé aux États-Unis et travaillant pour le Pentagone, affirme «Au vingt et unième [siècle], il y aura aussi deux calamités : l'islamisme radical, et l'anti-islamisme radical. ${ }^{79}$

Dans Le premier siècle après Béatrice, le rôle du prophète est décrit par l'entomologiste : "Le futur se trouve tout entier dans le présent, mais masqué, mais codé, mais en ordre dispersé. ${ }^{80}$ Mais ce ne sont que les élus, les rares élus,qui parviennent à déchiffrer le présent pour y voir l'avenir.

Les prophéties de Maalouf sont parfois plus saisissantes et risquent même de laisser tomber le lecteur dans la métaphysique. Dans Le premier siècle après Béatrice, toute l'intrigue commence à la place Tahrir. Ce même roman finit avec un Égyptien nommé Morsi, le même prénom du président élu bien des années après la publication de ce roman. Pure coïncidence ? C'est possible car après tout la place Tahrir est l'une des places les plus fréquentées de l'une des villes les plus populeuses au Moyen-Orient et le prénom Morsi n'est pas rare en Égypte.

\footnotetext{
${ }^{77}$ BOUVET, Rachel et EL KETTANI, Soundous, Op.cit., p.151

${ }^{78}$ Ibid., p.149

${ }^{79}$ Les Désorientés, p.503.

${ }^{80}$ Le premier siècle après Béatrice, p. 36.
} 


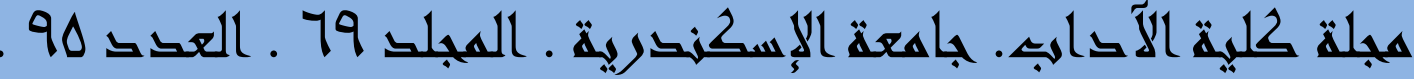

Cette clairvoyancepar laquelle nous pouvons qualifier l'œuvre de Maalouf lui a permis d'imaginer en 1992 un groupe de médecins indiens qui se réunissent pour dénoncer publiquement les pratiques de sexo-sélection dans leur pays et de prévoir en 1998 une attaque terroriste sur un symbole de l'hégémonieaméricaine et l'embrasement des banlieues françaises. Elle lui permet même de «penser qu'un jour, un Noir sera éluprésident des ÉtatsUnis. " ${ }^{81}$

Cette clairvoyance peut, en effet, s'expliquer par «les assises historiques ${ }^{82}$ de l'œuvre.Il est certain que l'expériencede Maalouf dans l'analyse des mouvements de l'Histoire influence grandement sa perspective de l'avenir. Tel qu'il l'affirme lui-même : «L'histoire, pour moi, n'est pas que pour l'histoire, le passé pour le passé. Il s'agit toujours de préoccupations liées à aujourd'hui, aux questions de coexistence [...]. ${ }^{83} \mathrm{La}$ fonction de l'historien vient accompagner la fonction du prophète. Ce jeu d'échos entre les réminiscences de l'Histoire et les visions du futur résonne partout dans son œuvre.

En conclusion, notre analyse des Identités meurtrières nous a permis de distinguer les axes principaux de la pensée de Maalouf. Son analyse de l'identité lui a permis de comprendre pourquoi et comment l'identité devient-elle meurtrière. Elle lui a permis en même temps de trouver la solution dans l'acceptation de la pluralité. Les déchaînements identitaires dans le monde entier ne sont que le résultat des attitudes intolérantes et partiales. Toute son œuvre est un hymne à la diversité et au respect de tous les hommes appartenant à toutes les cultures et les civilisations.

Peut-on dire que les propos de Maalouf sont des évidences consensuelles ? Il semblerait que oui. Cependant, et malheureusement, les tensions persistent et s'aggravent. Ces vérités ne sont donc « ni suffisamment évidentes, ni intimement reconnues. ${ }^{84}$

\footnotetext{
${ }^{81}$ Les Identités meurtrières, p.179

${ }^{82}$ BOUVET, Rachel et EL KETTANI, Soundous, Op.cit., p.151.

${ }^{83}$ Ibid., p.197

${ }^{84}$ Les Identités meurtrières, p.51
} 


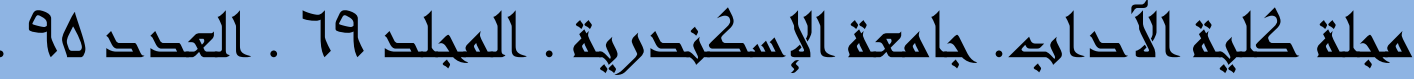

Le lecteur ne peut manquer de se demander : la pensée de Maalouf, est-elle une utopie ou un espoir ? L'actualité du monde ne nous fait-elle pas sourire devant son optimisme ? Son œuvre pourrait-elle changer notre actualité sombre et triste?

«Je ne sais pas si l'œuvre d'un écrivain influe sur son temps. Pas beaucoup, me semble-t-il, et pas à court terme. Surtout à notre époque, qui est à la fois la meilleure et la pire : la meilleure, parce qu'on peut s'y exprimer librement, et diffuser très largement ce que l'on dit; et la pire, parce que tout ce qui est dit se perd dans un gigantesque brouhaha planétaire, et finit par ne produire aucun effet. Néanmoins, on a le devoir de dire ce que l'on pense, ce que l'on prévoit, ce que l'on redoute. Que cela produise un effet ou pas. ${ }^{85}$

Maalouf écrit à partir de son expérience personnelle avec cette sensibilité et cette humanité qui font de lui un auteur exceptionnel. Avant de prêcher la coexistence entre les différentes cultures, il a su lui-même assumer toutes ses appartenances.

Son raisonnement nous mène à comprendre que ce n'est en respectant lesdifférences, dans un monde de plus en plus pluraliste qu'on pourra atteindre le salut de l'humanité. Choisir de refouler l'autre ne fait qu'embraser le monde dans une violence sans fin. En effet, ce qui entrave le dialogue, c'est moins la quête de la vérité que la prétention de ceux qui croient la posséder une fois pour toutes. Voici le centre de toute la pensée de Maalouf.

Lorsque l'identité devient le refuge pour faire face aux dangers, il est normal qu'elle devienne meurtrière. Blessé, marginalisé, exploité, atteint dans son propre orgueil, le monde musulman brandit haut et fort son identitéreligieuse en exaltant les moments d'un passé glorieux pour faire face à un Occident dominant politiquement et économiquement. L'inégalité n'a pas de religion. Si les pays musulmans manifestent de la rancune à l'égard de l'Occident, c'est à cause de la pauvreté et de la domination.

Amin Maalouf est profondément convaincu que l'islam est capable de se moderniser au même titre que le christianisme si la modernisation

\footnotetext{
${ }^{85}$ BOUVET, Rachel et EL KETTANI, Soundous, Op.cit.,p.p. 261, 262.
} 


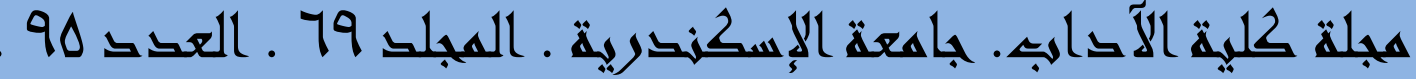

parvient à s'opérer dans les sociétés musulmanes en premier lieu. Si le monde arabo-musulman s'enlise dans la spirale de la violence, c'est que sa rancœur est contre lui-même d'abord avant d'être contre l'Occident.

Dix ans après la parution desIdentitésmeurtrières, Maalouf tiendra les mêmes propos dans Le Dérèglement du monde. La situation n'est que plus complexe et plus difficile. Cependant, l'auteur ne décourage point. Il affirme que ce sont les éclairés qui parviendront à briser ce cloisonnement et invite tout être humain à assumer ses appartenances multiples dans la sérénité, sans déchirements, puisqu'il y va de l'avenir et de la paix des sociétés humaines.

Sans doute, Maalouf ne propose-t-il pas de solutions magiques pour venir à bout des problèmes. Ses idées pourront cependant rendre la vie moins crispée et moins violente car il est à la recherche de vraies solutions. Or ce n'est qu'en posant un regard différent sur la notion d'identité, qu'on pourrait contribuer à trouver un chemin hors de l'impasse, un chemin capable de garantir les droits de la personne humaine, de toute personne humaine indépendamment de leur religion, de leur couleur et de leur nationalité.

Dans un monde où les idées sont de plus en plus brouillées, déformées par les médias et les intérêts économiques et politiques, la clairvoyance de Maalouf enchante son lecteur. Si ses propos sont ceux d'un migrant et d'un minoritaire, c'est justement ce qui fait toute sa richesse et sa particularité. Dans sa réponse au discours de réception d'Amin Maalouf à l'Académie française, le 14 juin 2012, Jean-Christophe Rufin relève cette particularité en s'adressant à Maalouf: "Nous vous accueillons tout entier et sans réserve, multiple comme vous l'êtes. Et nous vous disons, avec une affection [...] : prenez place parmi nous. Entrez ici avec 'vos noms, vos langues, vos croyances, vos fureurs, vos égarements, votre encre, votre sang, votre exil". Devenez dès cet instant l'un des nôtres, mais surtout, Monsieur, restez vousmême. ${ }^{86}$

Laissons, enfin, la parole à Maalouf car personne, mieux que lui, ne mettrait fin à cet article. Écoutons-le à la fin de son essai :

\footnotetext{
${ }^{86}$ MAALOUF, Joseph, Amin Maalouf. Itinéraire d'un humaniste éclairé, L'Harmattan « Coll. Pensée religieuse \& philosophique arabe », 2014, p.225.
} 


\title{
هجلة حلية الآثايت. باهعة الإسكنـرية ـ المجلى 79 ـ العقد 90.
}

«D'ordinaire, lorsqu'un auteur arrive à la dernière page, son vou le plus cher est que son livre soit encore lu dans centans, dans deux cent ans. [...]

Pour ce livre, qui n'est ni un divertissement ni une auvre littéraire, je formulerai le væu inverse: que mon petit-fils devenu homme, le découvrant un jour dans la bibliothèque familiale, le feuillette, le parcoure un peu, puis le remette aussitôt à l'endroit poussiéreux d'où il l'avait retiré, en haussant les épaules, et en s'étonnant que du temps de son grand-père, on eut encore besoin de dire ces choses-là. ${ }^{87}$

\author{
Amany GHANDER
}

Département de langue et de littérature françaises

Faculté des Lettres

Université d'Alexandrie

${ }^{87}$ Les Identités meurtrières, p.p. 188,189 